\title{
Полупроводниковая лазерная квазирешетка с фазированными одномодовыми каналами излучения
}

\author{
(C) Н.Ю. Гордеев ${ }^{1}$, А.С. Паюсов ${ }^{1}$, М.В. Максимов ${ }^{2}$ \\ ${ }^{1}$ Физико-технический институт им. А.Ф. Иоффе Российской академии наук, \\ 194021 Санкт-Петербург, Россия \\ ${ }^{2}$ Санкт-Петербургский национальный исследовательский Академический университет Российской академии наук, \\ 194021 Санкт-Петербург, Россия \\ E-mail: nkt.grdv@gmail.com
}

Поступила в Редакцию 15 апреля 2019 г.

В окончательной редакции 22 апреля 2019 г.

Принята к публикации 22 апреля 2019 г.

Предложена конструкция полупроводникового лазера с полосковым волноводом, геометрия которого может позволить получить излучение, аналогичное излучению фазированной лазерной решетки. Обсуждаются требования к параметрам лазерной квазирешетки и технологическая реализуемость предлагаемого подхода.

Ключевые слова: диодный лазер, фазированная лазерная решетка, оптический волновод, диаграмма направленности.

DOI: 10.21883/FTP.2019.10.48305.9140

\section{1. Введение}

Торцевые одиночные полупроводниковые лазеры полосковой геометрии условно можно разделить на две группы: пространственно-одномодовые лазеры с шириной полоскового волновода в единицы микрон, излучающие оптическую мощность в пределах 1 Вт, и мощные лазеры, которые за счет расширенного полоска позволяют получать мощность до десятков ватт при непрерывной накачке. Существенным недостатком последних является многомодовый характер излучения в горизонтальном направлении (вдоль плоскости $p-n$-перехода), что сильно ухудшает качество лазерного пучка и создает трудности при его фокусировке. Получение оптической мощности более 20 Вт возможно при использовании лазерных линеек или решеток, представляющих собой по сути массив лазеров с широким полосковым волноводом, изготовленный на одном полупроводниковом кристалле. Лазеры в такой линейке излучают независимо друг от друга, и полная интенсивность линейки является суммой интенсивностей отдельных ее элементов. Особый интерес представляет вариант линейки синфазно излучающих лазеров. Ее принцип действия основан на хорошо известном эффекте конструктивной интерференции ансамбля синфазных идентичных гармонических осцилляторов. В идеальных условиях, в результате интерференции ширина лазерного пучка будет обратно пропорциональна общей апертуре лазерной линейки, а угловая интенсивность в максимуме будет равна интенсивности одиночного лазера, умноженной на квадрат числа лазеров в линейке. Очевидно, что фазированная лазерная линейка должна конструироваться на основе массива пространственно-одномодовых лазеров. Поэтому в общем варианте лазерная линейка состоит из близко расположенных одномодовых лазеров, фазировка которых достигается за счет пространственного перекрытия их оптических полей [1].

Диаграмма излучения фазированной линейки в горизонтальном направлении при упрощенном анализе имеет основные и дополнительные максимумы, аналогично диаграмме, формируемой при дифракции Фраунгофера плоской монохроматичной волны на массиве периодических щелей. Однако уже в первых экспериментах по созданию фазированных лазерных решеток было показано, что их диаграмма направленности (дальнее поле излучения) существенно отличается от ожидаемой, что связано с принципиальным отличием механизма формирования диаграммы направленности лазерной линейки от простой дифракции плоской волны. Перекрытие оптических полей $N$ близко расположенных одномодовых лазеров приводит к формированию набора коллективных супермод, число которых равно $N$ [2]. При этом действительно одна из мод имеет дальнее поле, аналогичное интерференционной картине плоской волны. Остальные же моды практически эквивалентны интерференции $N$ излучателей с различной комбинацией разниц фаз 0 и $\pi$. Отмечалось, что есть тенденция лазерной генерации на так называемой осциллирующей моде, в которой любые два соседних лазера имеют разницу фаз $\pi$ [3]. Для дальнего поля излучения этой моды характерно наличие двух интенсивных максимумов, расположенных симметрично относительно нормали. В целом формирование супермод приводит к необходимости разработки методов их селективного подавления для преимушественной лазерной генерации одной выделенной моды. Эта проблема является основной при создании фазированной лазерной линейки. Для решения предлагались различные подходы, например встроенный фильтр Тальбо [4], антиволноводные лазерные решетки [5], Y-образные волноводы [6], интегрированные брэгговские решетки [7] и др. Однако 
большое число супермод и малое различие для них в эффективных показателях преломления, оптических потерях и пространственном распределении пока не позволили найти эффективного подхода.

В настоящей работе мы обсуждаем вариант полупроводникового лазера с полосковым волноводом, конструкция которого может позволить получить излучение, аналогичное излучению фазированной лазерной решетки. Также мы рассматриваем технологическую реализуемость предлагаемого подхода.

\section{2. Конструкция и параметры полупроводниковой лазерной квазирешетки с фазированными каналами излучения}

Основной принцип, лежащий в предлагаемой конструкции лазерной решетки, состоит в отсутствии супермод. Как отмечалось выше, эти моды образуются естественным образом в системе оптически связанных пространственно-одномодовых лазеров. Но перекрытие полей лазеров является необходимым условием для их фазировки. Наша идея состоит в использовании полоскового волновода, имеющего зигзагоподобную форму (рис. 1) с особыми параметрами. Для наглядности мы будем рассматривать конструкцию на примере лазеpa c активной областью на InGaAs квантовой яме (длина волны излучения $980 \mathrm{Hм}$ ) и $\mathrm{GaAs} / \mathrm{Al}_{0.15} \mathrm{Ga}_{0.85} \mathrm{As}-$ волноводом. Ширина полоска $W$ должна поддерживать существование только одной поперечной моды. Типичное значение $W$ составляет $\sim 4$ мкм и определяется глубиной травления полоскового волновода. Расстояние между $N$ полосками (период $d$ ) должно быть таким, чтобы оптические поля соседних полосковых волноводов не перекрывались. Это расстояние также зависит от глубины травления полоска и толщины вертикального волновода и может составлять величину в единицы микрон [3]. Вблизи граней лазерного кристалла полоски плавно изгибаются и подходят к зеркалам под углом. У одной из граней этот угол должен превышать угол

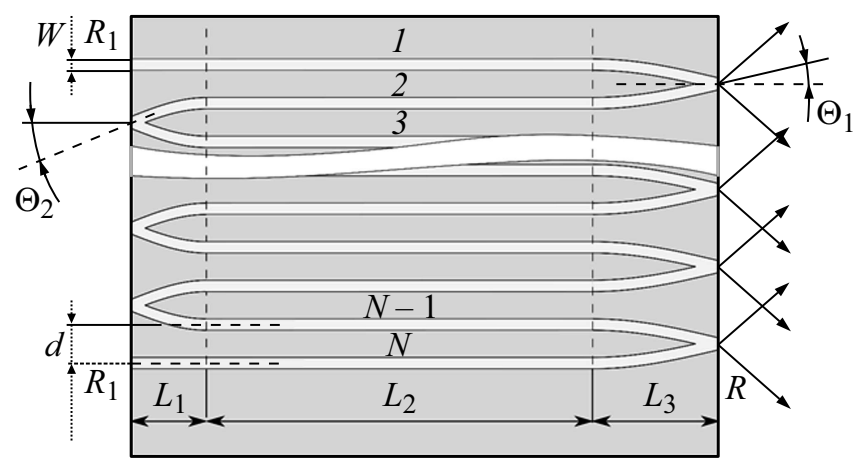

Рис. 1. Схематическое изображение лазерной квазирешетки (вид сверху). полного внутреннего отражения (ПВО):

$$
\Theta_{2}=\arcsin \left(1 / n_{\mathrm{eff}}\right),
$$

где $n_{\text {eff }}$ - эффективный показатель преломления волноводной моды, который для рассматриваемой системы равен $\sim 3.5$, что дает величину $\Theta_{2} \approx 16.5^{\circ}$. Свет отражается от зеркала и распространяется дальше по волноводу. Вблизи другой грани угол наклона полоска $\Theta_{1}$ выбирается меньше угла полного внутреннего отражения, что позволяет частично выводить излучение наружу. Число таких излучающих элементов, как видно из рис. 1 , равно $N / 2$. Коэффициент отражения рассчитывается по формулам Френеля для ТЕ-моды, типичной для полосковых лазеров:

$$
R=\left[\frac{\left(n_{\mathrm{eff}} \cos \Theta_{1}-\sqrt{1-n_{\mathrm{eff}}^{2} \sin ^{2} \Theta_{1}}\right)}{\left(n_{\mathrm{eff}} \cos \Theta_{1}+\sqrt{1-n_{\mathrm{eff}}^{2} \sin ^{2} \Theta_{1}}\right)}\right]^{2}
$$

$R$ в зависимости от угла изменяется от 0.31 (нормальное падение) до 1 (ПВО). Например, при $\Theta_{1}=10$ град $R \approx 0.39$. Важным в конструкции квазирешетки является наличие зеркал $R_{1}$, нормальных к оси волновода, имеющих отражение $R_{1} \approx 0.31$. За счет этих зеркал обеспечивается встречное распространение волн, аналогично простому резонатору Фабри-Перо.

Рассмотрим величину выходных оптических потерь $\alpha_{\text {out. }}$ Полная длина резонатора составляет $N \cdot L$, где $L \approx L_{1}+L_{2}+L_{3}$ (рис. 1). Для полного обхода резонатора будет справедливо выражение

$$
\exp \left(\alpha_{\text {out }} 2 L N\right)=(1 / R)^{N} \cdot\left(1 / R_{1}\right)^{2},
$$

в котором учтено, что при полном обходе есть $N$ зеркал с отражением $R$, два зеркала с отражением $R_{1}$ и $N$-2 зеркала с отражением 1 . После простого преобразования (3) получаем

$$
\alpha_{\text {out }}=1 / 2 L \ln \left[1 /\left(R \cdot R_{1}^{2 / N}\right)\right] .
$$

При увеличении $N$ коэффициент $R_{1}^{2 / N}$ стремится к 1 , а $\alpha_{\text {out }}$ - к выходным оптическим потерям обычного полоскового лазера длиной $L$ с зеркалами 1 и $R$. При этом есть возможность несколько уменьшить $\alpha_{\text {out }}$, нанеся на грань с зеркалами $R_{1}$ диэлектрическое отражающее покрытие. В этом случае нужно скорректировать угол $\Theta_{2}$, чтобы выполнялось условие ПВО.

Кроме оптических потерь на выход излучения и внутренних потерь, обычных для полоскового лазера, в квазирешетке присутствуют еще специфические оптические потери, обусловленные изгибом волновода (области $L_{1}$ и $L_{3}$ на рис. 1$)$, которые в литературе также называют радиационными потерями [8]. Их величина растет экспоненциально с уменьшением радиуса изгиба волновода. Тем не менее существует ряд фотонных приборов, в 


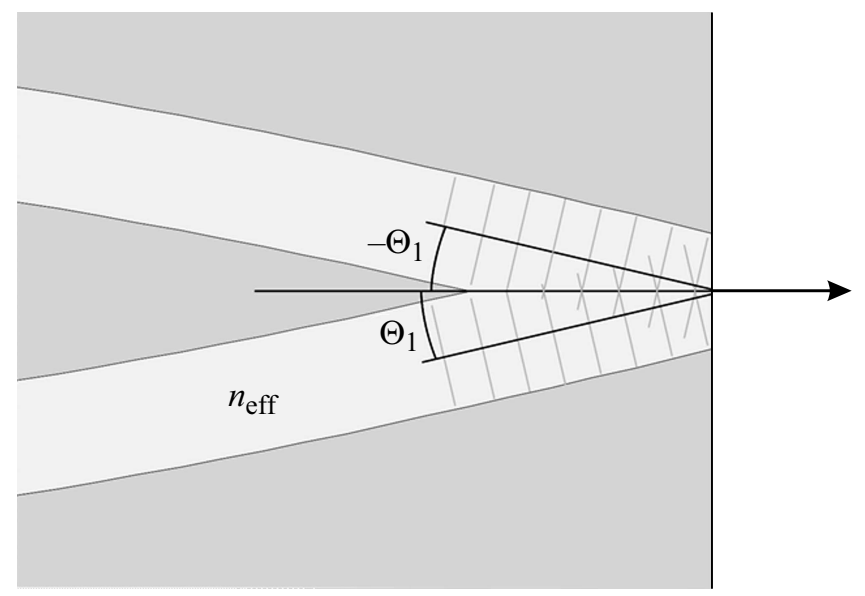

Рис. 2. Схематическое изображение одиночного излучающего элемента квазирешетки.

которых изогнутый волновод является важнейшим конструктивным элементом, например, суперлюминесцентный диод [9]. Подходом, позволяющим заметно уменьшить уровень радиационных потерь, является увеличение контраста полоскового волновода до величин более 0.01. Для обеспечения этой возможности вертикальный волновод должен быть достаточно тонким, $\sim 300$ нм для системы GaAs/AlGaAs [9]. Применительно к квазирешетке высококонтрастный полосковый волновод имеет еще дополнительные преимущества. Он сильнее ограничивает поперечную горизонтальную моду, тем самым позволяя уменьшить расстояние между соседними полосками без опасности возникновения оптической связи между ними [3]. Более плотное расположение полосков и излучательных областей на выходном зеркале предпочтительно для дальнего поля излучения, которое мы обсуждаем далее. Отметим, что изогнутый волновод имеет и положительный эффект, поскольку радиационные потери возрастают для горизонтальных мод более высоких порядков. Это значит, что для квазирешетки можно использовать полосковый волновод большей ширины, а возникающая горизонтальная мода высокого порядка будет подавляться при распространении по изогнутому волноводу [10].

Практический интерес представляет диаграмма направленности, формируемая квазирешеткой. Детальный анализ требует рассмотрения по типу дифракции Фраунгофера наклонных волн на широких щелях с учетом отражения от наклонного зеркала [11] и выходит за рамки данной статьи. Мы ограничимся упрощенным рассмотрением. На рис. 2 представлен элемент квазирешетки, из которого свет выводится наружу. Волны по полоску распространяются во встречных направлениях, образуя подобие стоячей волны, что обеспечивается зеркалами $R_{1}$. Это отличает квазирешетку от, например, кольцевых лазеров [12], в которых есть тенденция преимушественного распространения света в одном направ- лении по часовой стрелке или против нее, что приводит к специфической картине дальнего поля излучения с максимумом, расположенным под большим углом к выходному зеркалу. В предлагаемой квазирешетке на зеркало $R$ падают две волны одинаковой амплитуды под углами $\pm \Theta_{1}$, образуя на зеркале интерференционные полосы с расстоянием между максимумами:

$$
\Delta x=\lambda /\left(2 n_{\mathrm{eff}} \sin \Theta_{1}\right),
$$

где $\lambda-$ длина волны излучения. Подставляя в (5) численные значения модельной системы, получаем $\Delta x \approx 0.8$ мкм - величину порядка длины волны. Таким образом, за счет большого угла $\Theta_{1}$ ближнее поле излучения на выходном зеркале будет достаточно однородным. В силу угловой симметричности интерферирующих волн и малого $\Delta x$ в дальнем поле излучения одиночного элемента будет присутствовать один максимум, нормальный к поверхности зеркала. Поскольку по всей длине полоскового волновода распространяется одна и та же волна, в областях вывода излучения выполняется условие синфазности. Ансамбль излучающих элементов можно упрощенно рассматривать, используя формализм дифракционной решетки, имеющей период $2 d$, равный периоду излучающих областей (рис. 1). Тогда угловое расстояние $\Theta_{m}$ между основными максимумами в диаграмме будет определяться как

$$
\Theta_{m}=\arcsin (\lambda / 2 d) .
$$

Ширина максимума зависит от суммарной апертуры излучения квазирешетки.

\section{3. Технологическая реализуемость предлагаемого подхода}

Предлагаемая квазирешетка может быть создана в процессе постростовой технологической обработки эпитаксиальной лазерной структуры. Изготовление полоскового волновода осуществляется с использованием стандартных методов фотолитографии. Для травления структуры целесообразно использовать плазмохимическое травление, которое позволяет травить на большую глубину и получать практически вертикальные стенки. Критическим моментом является формирование выходных зеркал квазирешетки. Обычно зеркала торцевых диодных лазеров и подобных приборов (суперлюминесцентные диоды, оптические усилители) формируются естественными гранями полупроводникового кристалла при его скалывании. При этом точность расположения грани очень мала, поэтому такой подход невозможно использовать для предлагаемой квазирешетки, в которой взаимное расположение наклонных полосков и зеркал является важнейшей составляющей. Проблема может быть решена, если использовать технологию травления зеркал. Было показано [13], что технологии травления позволяют делать зеркала с малой шероховатостью, 
лишь незначительно ухудшающей характеристики (порог, дифференциальная квантовая эффективность) лазеров с узким полосковым волноводом. Использование при этом фотолитографии дает возможность прецизионно располагать зеркала относительно других конструктивных элементов лазера. В квазирешетке шероховатость поверхности зеркал будет играть еще меньшую роль, поскольку свет падает под углом к поверхности. Характеристики реального прибора будут неизбежно зависеть от естественного технологического отклонения параметров.

\section{4. Заключение}

Предложена конструкция полупроводникового лазера с полосковым пространственно-одномодовым волноводом, расположенным зигзагообразно на гетероструктуре. Расстояние между полосками таково, что оптические поля соседних полосковых волноводов не перекрываются. За счет этого в предлагаемой конструкции лазерной решетки отсутствуют супермоды, характерные для традиционных лазерных решеток на основе нескольких лазеров. За счет изгиба полосков на одной из граней решетки выполняется условие полного внутреннего отражения падающего света, а излучение выводится с противоположной грани через периодически расположенные излучающие области. Дальнее поле излучения квазирешетки проанализировано с использованием формализма дифракционной решетки. Показано, что существующие технологии позволяют реализовать на практике предложенную конструкцию лазерной квазирешетки.

\section{Благодарности}

Автор А.С.Паюсов благодарит Российский научный фонд за поддержку (проект 17-72-10060).

\section{Конфликт интересов}

Авторы подтверждают отсутствие конфликта интересов.

\section{Список литературы}

[1] D. Botez. IEEE J. Quant. Electron., 21 (11), 1752 (1985).

[2] E. Kapon, J. Katz, A. Yariv. Optics Lett., 9 (4), 125 (1984).

[3] V.P. Chaly, S.Y. Karpov, A.L. Ter-Martirosyan, D.V. Titov, W.Z. Guo. Semicond. Sci. Technol., 11 (3), 372 (1996).

[4] D. Mehuys, W. Streifer, R.G. Waarts, D.F. Welch. Optics Lett., 16 (11), 823 (1991).

[5] A.P. Napartovich, N.N. Elkin, V.N. Troshchieva, D.V. Vysotsky, L.J. Mawst, D. Botez. IEEE J. Select Top. Quant. Electron., 17 (6), 1735 (2011).

[6] D. Welch, P. Cross, D. Scifres, W. Streifer, R.D. Burnham. Electron. Lett., 22 (6), 293 (1986).

[7] Y. Zhao, L. Zhu. Opt. Express, 20 (6), 6375 (2012).

[8] E.A.J. Marcatili, S.E. Miller. Techn. J., 48 (7), 2161 (1969).
[9] Q. An, P. Jin, J. Wu, Z. Wang. Semicond. Sci. Technol., 27 (5), 055003 (2012).

[10] R.B. Swint, T.S. Yeoh, V.C. Elarde, J.J. Coleman, M.S. Zediker. IEEE Photon. Techn. Lett., 16 (1), 12 (2004).

[11] D. Marcuse. J. Light. Technol., 7 (2), 336 (1989).

[12] M.F. Booth, A. Schremer, J.M. Ballantyne. Appl. Phys. Lett., 76 (9), 1095 (2000).

[13] G. Adolfsson, J. Bengtsson, Å. Haglund, B. Nilsson, A. Larsson. J. Appl. Phys., 109 (9), 093112 (2011).

Редактор Г.А. Оганесян

\section{Semiconductor laser quasi-array with phase-locked single-mode emitting channels}

\author{
N.Yu. Gordeev ${ }^{1}$, A.S. Payusov ${ }^{1}$, M.V. Maximov ${ }^{2}$ \\ ${ }^{1}$ loffe Institute, \\ 194021 St. Petersburg, Russia \\ 2 St. Petersburg Academic University, \\ 194021 St. Petersburg, Russia
}

\begin{abstract}
We have proposed a semiconductor laser design with a stripe waveguide, which geometry can make it possible to obtain radiation similar to that of a phase-locked laser array. The requirements for the laser quasi-array parameters and the technological feasibility of the proposed approach are discussed.
\end{abstract}

